EPJ Web of Conferences 92,02088 (2015)

DOI: $10.1051 /$ epjconf/ 20159202088

(C) Owned by the authors, published by EDP Sciences, 2015

\title{
Investigation of flow in axial turbine stage without shroud-seal
}

\author{
Petr Straka ${ }^{1, a}$, Martin Němec ${ }^{1}$, Tomáš Jelínek ${ }^{1}$ \\ ${ }^{1}$ VZLU, a.s., Beranových 130, Prague-Letňany, Czech Republic
}

\begin{abstract}
This article deals with investigation of the influence of the radial gaps on the efficiency of the axial turbine stage. The investigation was carried out for the axial stage of the low-power turbine with the drumtype rotor without the shroud. In this configuration the flow through the radial gap under the hub-end of the stator blades and above the tip-end of the rotor blades leads to generation of the strong secondary flows, which decrease the efficiency of the stage. This problem was studied by experiment as well as by numerical modelling. The experiment was performed on the test rig equipped with the water brake dynamometer, torque meter and rotatable stator together with the linear probe manipulator. Numerical modelling was carried out for both the steady flow using the "mixing plane" interface and the unsteady flow using the "sliding mesh" interface between the stator and rotor wheels. The influence of the radial gap was studied in two configuration a) positive and b) negative overlapping of the tip-ends of the rotor blades. The efficiency of the axial stage in dependence on the expansion ratio, velocity ratio and the configuration as well as the details of the flow fields are presented in this paper.
\end{abstract}

\section{Introduction}

The axial steam turbines of low power (around hundreds kilowatts to units megawatts) are typically designed with the drum-type rotor as shown in figure 1. For reduction of a production cost the shroud-less stator and rotor wheels are still in use. In this configuration it is necessary to maintain the radial gap under the hub-end of the stator blades and above the tip-end of the rotor blades. However, flow through the radial gap generates large secondary flow structures which has negative influence on the efficiency. Influence of the secondary flow through the radial gap must be taken into account in the design of the blades. The available empirical informations (e.g. [1]) are often out of date or quite simplified. Therefore, research of the influence of the radial gap on flow in the axial turbine stages is still very actual because this kind of turbines are still in use. Currently, the experimental investigation (see $[2,3]$ ) as well as numerical methods (e.g. $[4,5])$ are gaining importance in research of the secondary flow in the turbine stages.

Presented work was carried out under the project: TA02021336 - Research and Development of Small Sized Turbine Stage (see [6]). Project deals with research of an aerodynamics and energy characteristics of the axial turbine stage. Attention is focused on the axial stage of an steam turbine of low power. The project includes two main objectives: 1) investigation of the aerodynamics and energy characteristics of the current design, 2) based on acquired knowledge to propose a new design. This paper deals with some results of the first objective. In section "Future work" some trends of solution of the second objective will be mentioned. The current design of the axial stage is based on the design methods of fifties of the last century. This design is characterized by use of a prismatic blades without shroud and seal.

\footnotetext{
a e-mail: straka@vzlu.cz
}

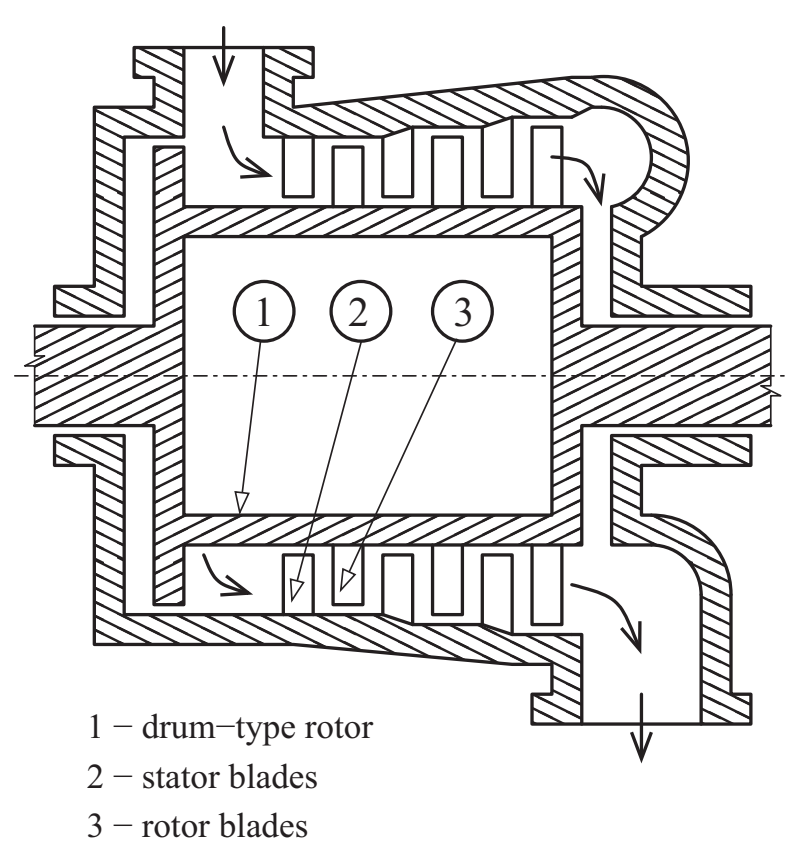

Fig. 1. Simplified scheme of axial turbine with drum-type rotor.

Both objectives of the project are solved through an experiment and numerical modelling. Used test facility as well as numerical software were developed at the highspeed laboratory of the aerodynamics department of the VZLU (see. [7]).

Figure 2 shows a scheme of the experimental stage. Contrary to the real turbine with the drum-type rotor, hubwalls of the experimental stage are non-rotating. Only the rotor blades are carried by a rotating disc.

The scheme in figure 2 shows also four defined planes (0 to 4 ) in which a parameters of flow field are investigated. Plane 0 lies $46 \mathrm{~mm}$ before the leading edge of stator blades, plane 1 lies in an axial gap between the stator and rotor 


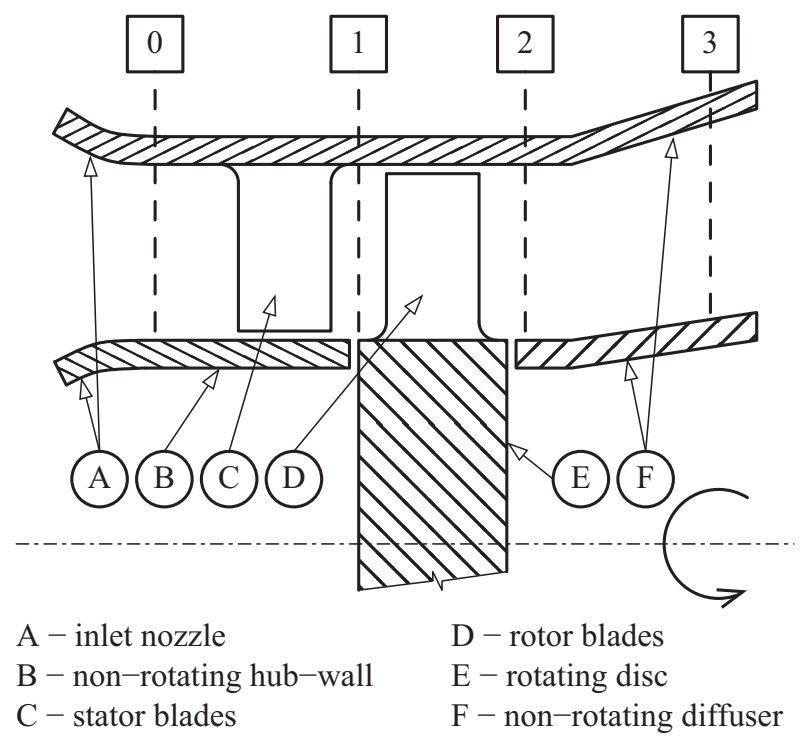

Fig. 2. Scheme of experimental stage.

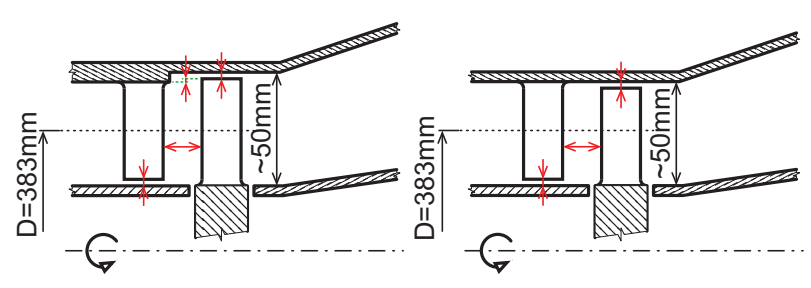

Fig. 3. Scheme of experimental stage, left: positive, right: negative overlapping of rotor blades tip-end.

wheels $8 \mathrm{~mm}$ behind the trailing edges of the stator blades, plane 2 lies $11 \mathrm{~mm}$ behind the trailing edges of the rotor blades and plane 3 lies $61 \mathrm{~mm}$ behind the trailing edges of the rotor blades.

Two configuration of overlapping of the tip-ends of the rotor blades (as shown in figure 3) are studied. In this work results for following dimensions of radial and axial gaps are presented: radial gap under the hub-end of the stator blades $1 \mathrm{~mm}$, radial gap above the tip-end of the rotor blades $1.4 \mathrm{~mm}$, size of positive overlapping $1 \mathrm{~mm}$ and axial gap between stator and rotor blades 8 and $15 \mathrm{~mm}$. Configuration with the axial gap between stator and rotor blades $15 \mathrm{~mm}$ was investigated both experimentally and numerically, while configuration with the axial gap $8 \mathrm{~mm}$ experimentally only.

For possibility to reduce computational cost in numerical modelling the experimental stage contains 50 stator and 60 rotor blades, however number of blades in real turbine is different with respect to dynamics properties.

\section{Test facility}

The test rig (shown in figure 4) is a part of the high-speed closed-loop wind tunnel powered by $1.3 \mathrm{MW}$ electro-engine. This facility allowing independent setting of the Mach and Reynolds numbers. A working level of pressure is maintained by a group of steady running vacuum pumps and by adjusting of an intake valve opening. The air sucked from atmosphere into the wind tunnel is passed through the draying plant to reduce the air humidity. The air supplied

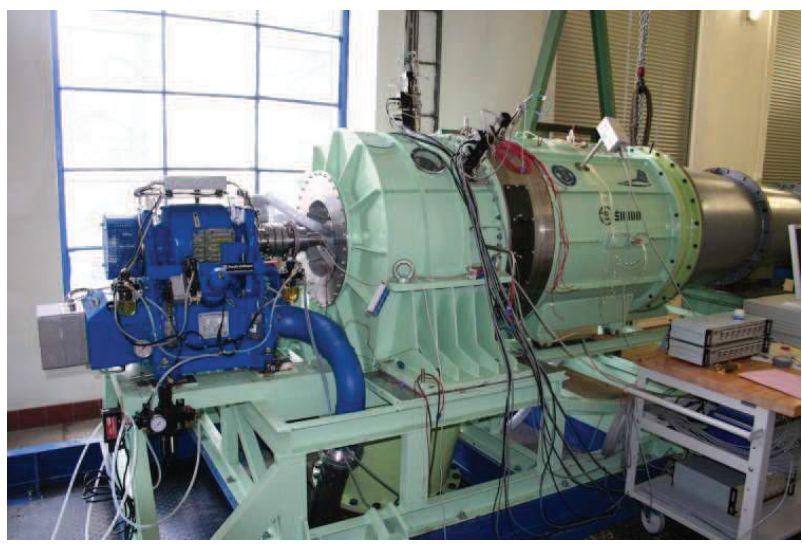

Fig. 4. Turbine test rig.

Table 1. Parameters of test rig.

\begin{tabular}{ll}
\hline Maximum rotational speed & $15000 \mathrm{rpm}$ \\
Mass flow rate & $6 \mathrm{~kg} \cdot \mathrm{s}^{-1}$ \\
Maximum brake power & $700 \mathrm{~kW}$ \\
Maximum expansion ratio & 8 \\
Accuracy of torque measurement & better then $\pm 0.5 \mathrm{Nm}$ \\
\hline
\end{tabular}

by a 13 th stage radial compressor is conducted throughout a pipe where the orifice place is mounted to measure the mass flow.

Tested turbine is loaded by hydraulic dynamometer Froude Hofmann F249-Race GT (water working fluid). Flange torque meter HBM T10FS is used for the connection of turbine's and dynamometer's shafts in order to increase the accuracy of the torque measurement. Parameters of the test rig are shown in table 1.

A concept of the rotatable stator was used in consequence of linear probe manipulators. This idea was motivated by several reasons which make the measurement and follow-up data analyses more convenient $[8,9]$. The probes were fitted in three linear motion mechanisms placed circumferentially along the casing with a separation angle of 60 degrees. Fast response pressure probes were also used in order to study the unsteady flow field at the outlet of the rotor. The probes design was based on Kulite transducers usage [10] and a method of virtual multi-hole probe [11, 12] was exploited in the measurement methodology.

\section{Numerical aparatus}

Flow through an axial turbine stage is modeled as $3 \mathrm{D}$, compressible, viscous, fully turbulent. Flowing medium is an perfect gas.

Two different interfaces between stator and rotor domain are used. Model of the unsteady interaction between stator and rotor uses the "sliding-mesh" interface, where communication between stationary stator domain and rotating rotor domain is via interpolation. Model of the quasisteady stator-rotor interaction uses the "mixing-plane" interface, where communication between stator and rotor domain is performed by weighted circumferential averaging.

The simulation is performed using the in-house numerical code based on solution of system of the RANS equation and the TNT $k-\omega$ turbulence model proposed by Kok 
[13]. Used numerical code $[14,15]$ is focused on turbomachinery application.

System of the governing equations written in vector form looks like:

$$
\frac{\partial \mathbf{W}}{\partial t}+\frac{\partial \mathbf{F}_{i}}{\partial x_{i}}-\frac{\partial \mathbf{F}_{i}^{v}}{\partial x_{i}}=\mathbf{Q},
$$

where $\mathbf{W}$ is vector of the conservative variables, $\mathbf{F}$ is vector of the inviscid fluxes, $\mathbf{F}^{v}$ is vector of the viscous fluxes and $\mathbf{Q}$ is vector of the source terms. System (1) is discretized by the cell-centered finite-volume method on multi-block structured mesh of hexahedral elements. Temporal discretization is performed using the second-orded backward Euler formula in implicit form, which is realized through a dual iterative process:

$$
\begin{gathered}
\left(\frac{3}{2} \mathbf{I}+\Delta t \frac{\partial \mathbf{R}_{i}^{n, v}}{\partial \mathbf{W}_{i}^{n, v}}\right) \Delta \mathbf{W}_{i}^{n, v+1 / 2}+\Delta t \sum_{j=1}^{6} \frac{\partial \mathbf{R}_{i}^{n, v}}{\partial \mathbf{W}_{j}^{n, v}} \Delta \mathbf{W}_{j}^{n, v+1 / 2}= \\
=-\frac{3 \mathbf{W}_{i}^{n, v}-4 \mathbf{W}_{i}^{n}+\mathbf{W}_{i}^{n-1}}{2}-\Delta t \mathbf{R}_{i}^{n, v}
\end{gathered}
$$

where $\mathbf{W}^{n, 0}:=\mathbf{W}^{n}, \Delta \mathbf{W}^{n, v+1 / 2}=\Delta \mathbf{W}^{n, v+1}-\Delta \mathbf{W}^{n, v}$ and $\mathbf{W}^{n+1}:=\mathbf{W}^{n, v^{*}}$, where $\mathbf{W}^{n, v^{*}}$ is a steady solution in the dual iterative process. In scheme (2) there $n$ means the time level, $v$ is index of the dual iteration and $\mathbf{R}$ is:

$$
\begin{aligned}
& \mathbf{R}_{i}=\frac{1}{V_{i}} \sum_{j=1}^{6}\left[\Phi^{i n v}\left(\mathbf{W}_{j}^{L}, \mathbf{W}_{j}^{R}, \mathbf{n}_{j}\right)\right. \\
& \left.-\Phi^{v i s}\left(\mathbf{W}_{j}^{C},(\nabla \mathbf{W})_{D_{j}^{\text {dual }}}, \mathbf{n}_{j}\right)\right] s_{j} .
\end{aligned}
$$

The inviscid numerical fluxes $\Phi^{i n v}$ are calculated using the exact solution of the 1D Rieman problem in normal direction to the cell edges. The viscous numerical fluxes $\Phi^{\text {vis }}$ are calculated using the central scheme and the gradient of the state vector $\nabla \mathbf{W}$ is calculated using the Green-Gauss theorem on a dual cells. Higher order of accuracy in space is obtained using linear reconstruction with the Van Leer's slope limiter.

\subsection{Computation setup}

The inlet and outlet boundary of the computational domain are located in planes 0 and 3 respectively (see figure 2). Using the model of the unsteady interaction between stator and rotor it is possible to better simulate flow in the axial stage than using model of the quasi-steady interaction. However the model of the unsteady interaction has higher demands on the size of the computational domain. It is necessary to use computational domain which contains periodical section of five stator and six rotor blades (as shown in figure 5), while the model of the quasi-steady interaction, via "mixing-plane", enables to reduce size of the computational domain to section which contains only one stator and one rotor blade.

The model of the quasi-steady interaction allows very reduce the computational cost but an disadvantage of this model is a physical simplification. Figure 6 shows structure of vortices which are generated consequently to flow through the radial gap under the hub-end of the stator blades. It is clear that application of the "mixing-plane" technique, which is based on the weighted circumferential averaging, leads to distortion of the vortices in some non-physical circumferential averaged shape.

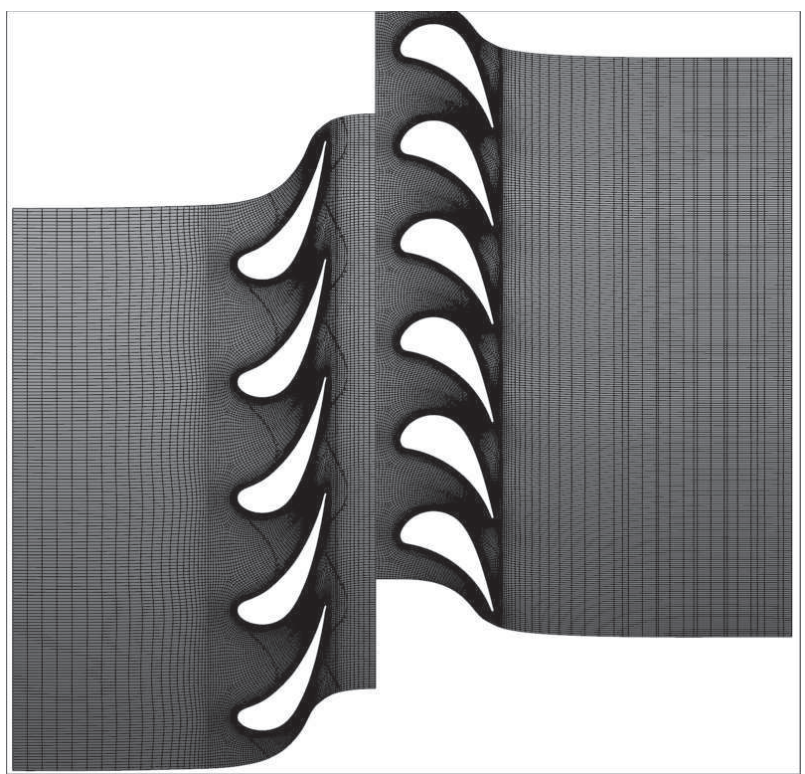

Fig. 5. Circumferential section of computational domain.

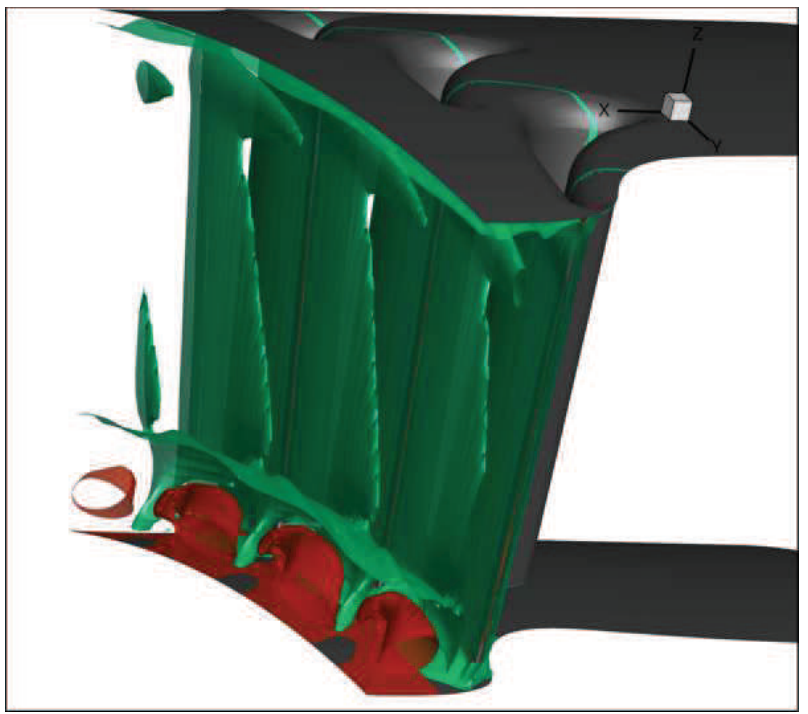

Fig. 6. Vortices behind the hub-end of the stator blades.

\section{Results}

Presented experimental results were measured in range of the total/static expansion ratio $\pi 0.35$ to 0.58 and in range of the velocity ratio $u / c_{2 i s} 1.6$ to 1.9 ( $u$ is the circumferential speed at the middle diameter, $c_{2 i s}$ is the isentropic velocity in plane 2). Numerical simulation was performed in range of the total/static expansion ratio $\pi 0.5$ to 0.6 and for two values of the velocity ratio $u / c_{2 i} 1.6$ and 1.9. All regimes in configuration with positive overlapping of the tip-end of the rotor blades were computed using the model of the quasi-steady interaction only, while all regimes in configuration with negative overlapping were computed using the model of the unsteady interaction. In addition, regimes with velocity ratio 1.6 in configuration with negative overlapping were also computed using the model of the quasi-steady interaction. It means that in some regimes it is possible to compare results of both models of the interaction between stator and rotor each other and also with experimental data, which is very useful. Figures 7 to 13 
shows comparison of span-wise distribution of some quantities in planes 1, 2 and 3.

In plane 1 distributions of the total pressure loss coefficient $\left(\zeta=1-p_{\text {tot } 1} / p_{\text {tot inlet }}\right)$ - figure 7 and the absolute Mach number $M_{a b s}$ - figure 8 are compared. Figure 7 shows rapidly increase of the total pressure loss in lower twenty percent of the span. It is an effect of the vortices which are generated behind the radial gap under the hubend of the stator blades (figure 6). The figure 7 shows that the model of the unsteady interaction predicts this phenomenon in good agreement with experiment, contrary the model of the quasi-steady interaction gives some differences compared to the experiment. In particular, the model of the quasi-steady interaction overestimates the height of the region influenced by mentioned vortices. It can be assumed that it is an effect of the "mixing plane" interface. Similar situation in lower twenty percent of the span we can see in figure 8 . In addition, figure 8 shows some overprediction of the absolute Mach number by both models of the interaction in upper fifteen to twenty percent of the span. It affects the prediction of the flow around the tip of the rotor blades as will be shown later.

In plane 2 the distributions of the total-total efficiency $\eta_{T T}$ - figure 9, the total-static efficiency $\eta_{T S}$ - figure 10 and the absolute Mach number $M_{a b s}$ - figure 12 are compared. Let us follow at first the distribution of the total-total and total-static efficiency in figures 9 and 10. It is obvious that in lower forty percent of the span the efficiency is around ten percent lower compared to value in sixty percent of the span. It is a consequence of an interaction of the hub part of the rotor blades with the vortices generated behind the radial gap under the hub-end of the stator blades. This vortices are drifted on the higher diameter along the suction side of the rotor blades as shown figure 11. From figures 9 and10 it is evident that the model of the unsteady interaction predicts mentioned interaction well, but the model of the quasi-steady interaction shows different distribution of the efficiency in lower part of the span. It is caused by the "mixing plane" interface. Contrary to the "sliding mesh" interface in case of the "mixing plane" no vortices interacts with the rotor blades but some un-physical structure, which arises by circumferential averaging. Little bigger difference of results obtained by both models of interaction compared to the experimental data is seen in the upper part of the span where an effect of the vortices generated behind the radial gap above the tip-end of the rotor blades is depicted. Both models under-predicts the height of the affected region and over-predicts decreasing of the efficiency in the affected region. Differences between the experimental data and results of the numerical modelling in the upper part of the span behind the rotor blades may be caused by differences of the Mach number in the axial gap between the stator and the rotor in upper part of the span as shown figure 8. Distribution of the absolute Mach number in figure 12 shows that results of the unsteady interaction model are near to the experimental data. While the model of the quasi-steady interaction overpredicts value of the absolute Mach number in the lower part of the span, which is above mentioned effect of the "mixing-plane" interface.

In plane 3 distributions of the absolute Mach number are compared in figure 13. It is seen that the model of the unsteady interaction better corresponds to the experimental data then the model of the quasi-steady interaction. How-
Table 2. Integral parameters in planes 1 and 2.

\begin{tabular}{r|cc|ccc}
\hline & \multicolumn{2}{|c|}{ plane 1 } & \multicolumn{3}{|c}{ plane 2 } \\
& $\zeta$ & $M_{a b s}$ & $\eta_{T T}$ & $\eta_{T S}$ & $M_{a b s}$ \\
\hline experiment & 0.34 & 0.58 & 0.791 & 0.743 & 0.20 \\
cfd unsteady & 0.39 & 0.58 & 0.802 & 0.748 & 0.22 \\
cfd quasi-steady & 0.45 & 0.59 & 0.815 & 0.748 & 0.25 \\
\hline
\end{tabular}

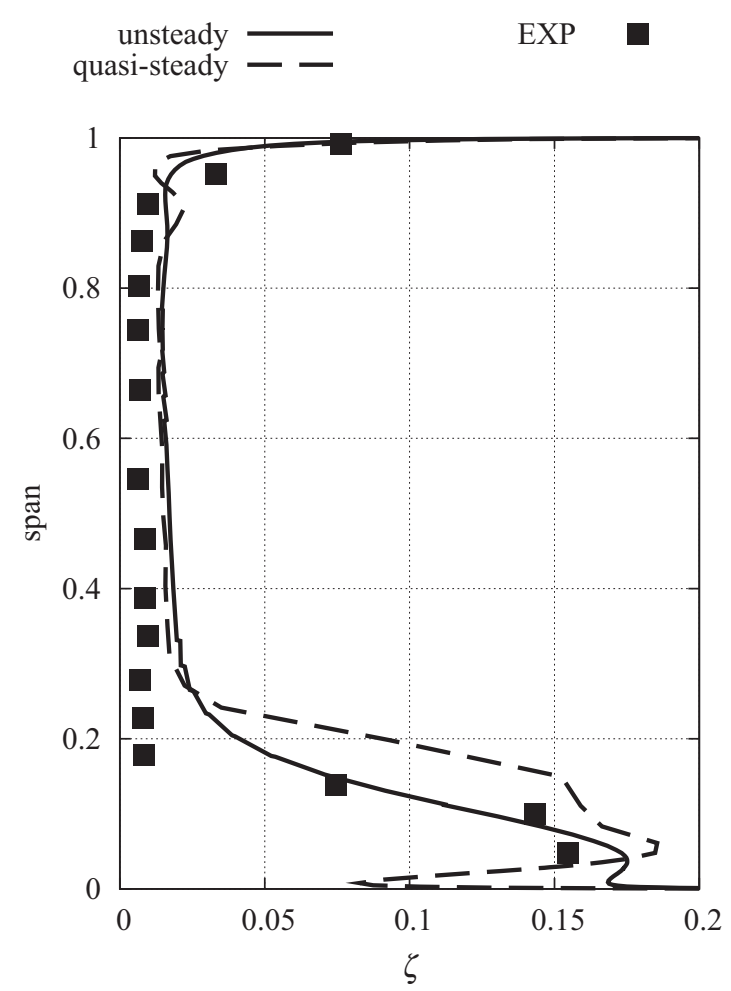

Fig. 7. Span-wise distribution of total pressure loss coefficient in plane 1.

ever in upper part of the span models predicts lower value of the absolute Mach number than shows the experiment.

In table 2 some integral parameters in planes 1 and 2 are compared. Although there are some differences in span-wise distribution of the total-total and total-static efficiency, the integral values are very similar.

In figures 14 and 15 the dependency of the total-static efficiency on the velocity ratio $u / c_{2 i s}$ is plotted for configuration with the negative (figure 14) and positive (figure 15) overlapping of the tip-end of the rotor blades. It is evident that numerical models predicts the efficiency about one to two percent higher compare to experimental data. It is also obvious that optimum of the velocity ratio considering to the efficiency is located out of range of the investigated regimes. The range of the measured and computed velocity ratios is based on the operating parameters of the real turbine, on the operating capabilities of the test rig and on the dynamical properties of the experimental stage. Note that the efficiency in figures 14 and 15 obtained via experiment were evaluated from static pressures measured on the huband tip-wall, while efficiency obtained via numerical modelling were evaluated as mass-averaged mean value from the span-wise distribution. 


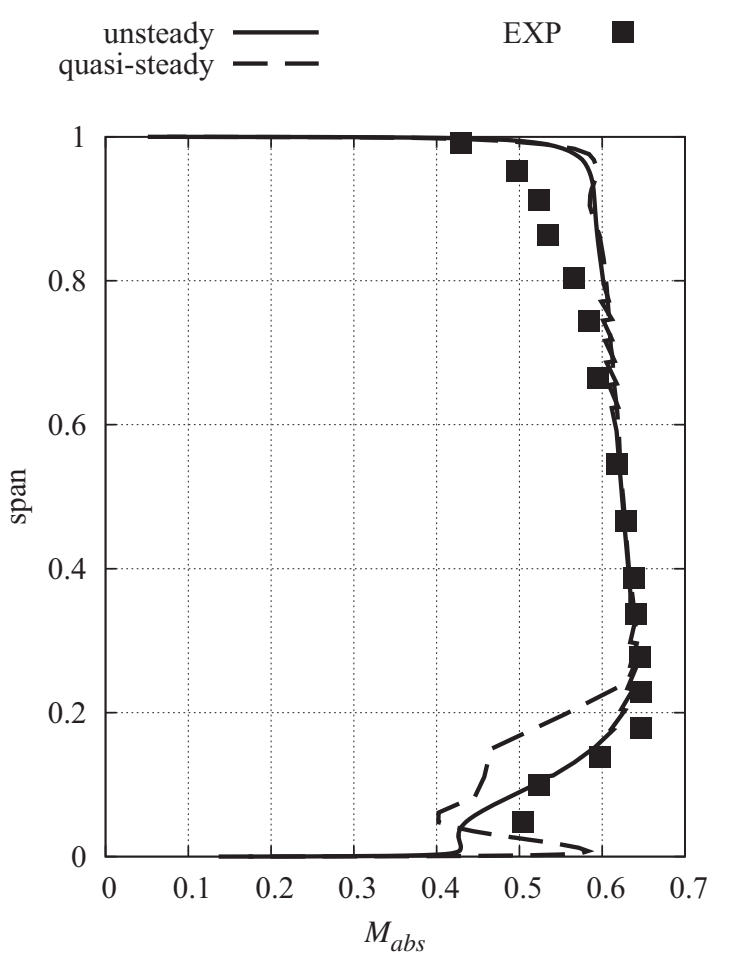

Fig. 8. Span-wise distribution of tbsolute Mach number in plane 1.

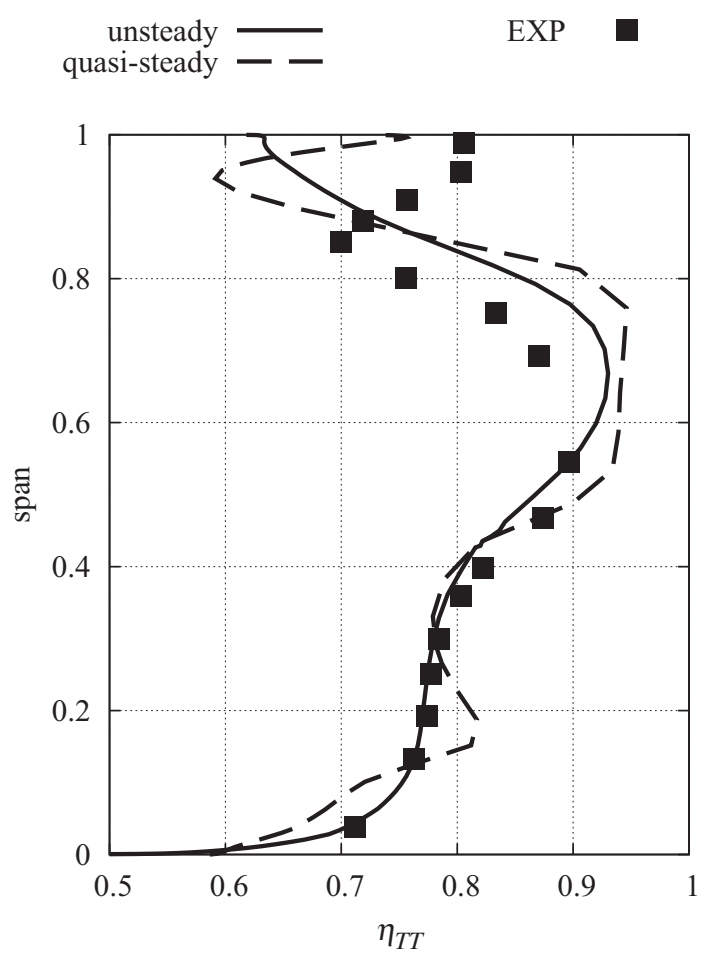

Fig. 9. Span-wise distribution of total-total efficiency in plane 2.

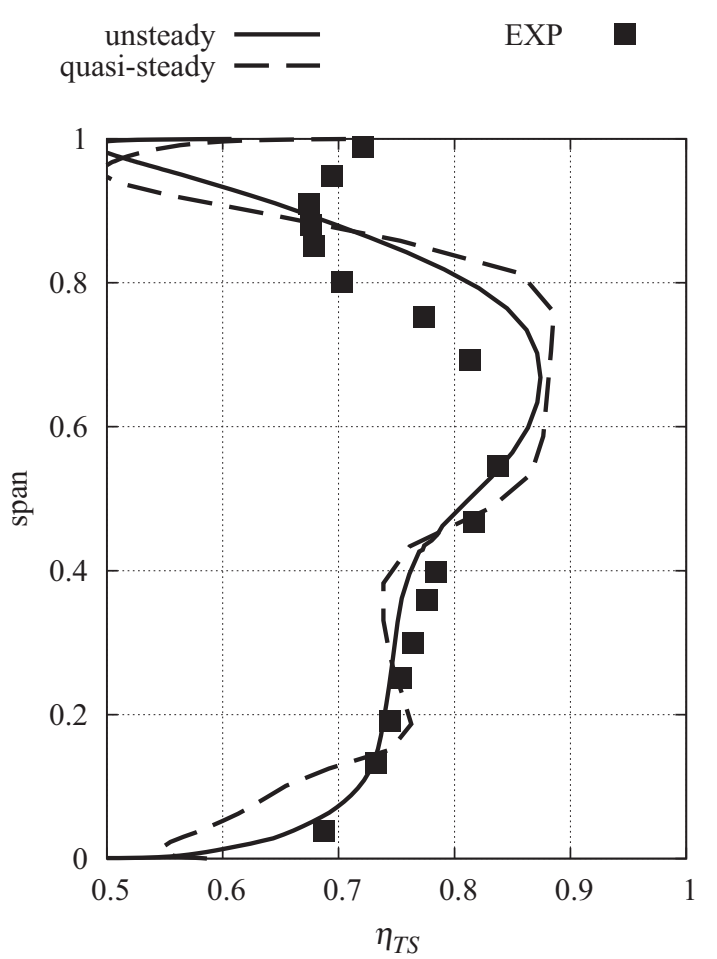

Fig. 10. Span-wise distribution of total-static efficiency in plane 2 .

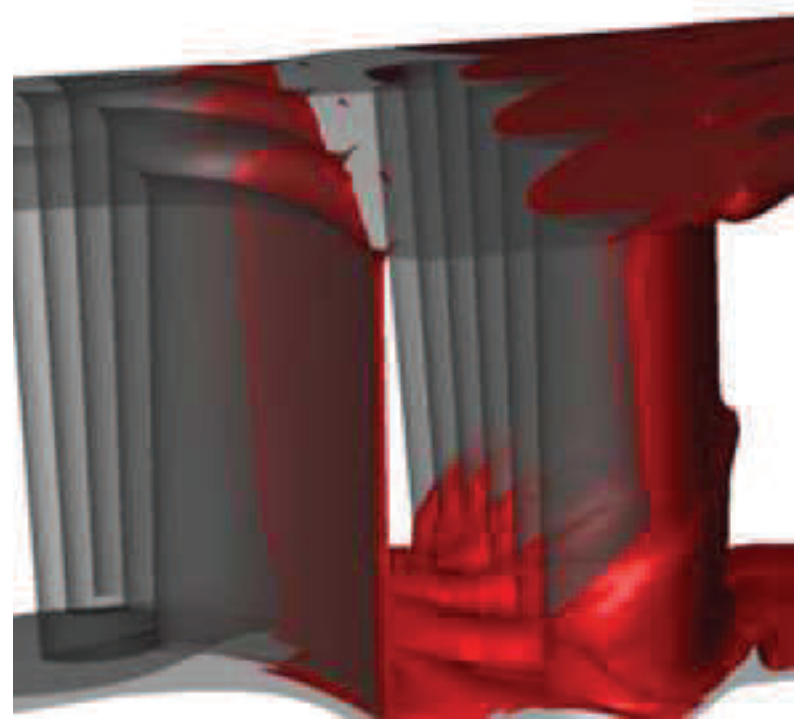

Fig. 11. Interaction of vortices (generated behind the radial gap under the hub-end of the stator blades) with the rotor blades.

\section{Conclusion}

Although comparison of the span-wise distribution of some quantities shows differences between experiment and results of the numerical modelling, detected differences are acceptable in technical practice.

Presented results shows that flow through the radial gaps under the hub-end of the stator blades and above tipend of the rotor blades has a strong effect on reducing of the efficiency of the turbine stage. Spanwise distribution of the efficiency in figures 9 and 10 shows that more than forty percent of the hub part of the span are affected by 


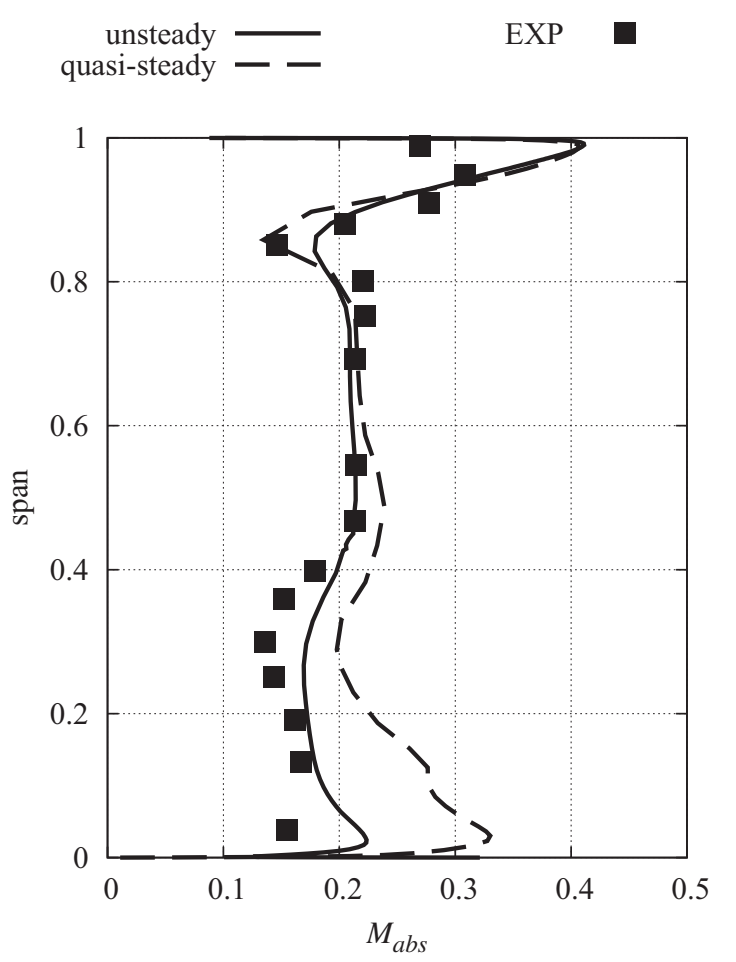

Fig. 12. Span-wise distribution of tbsolute Mach number in plane 2 .

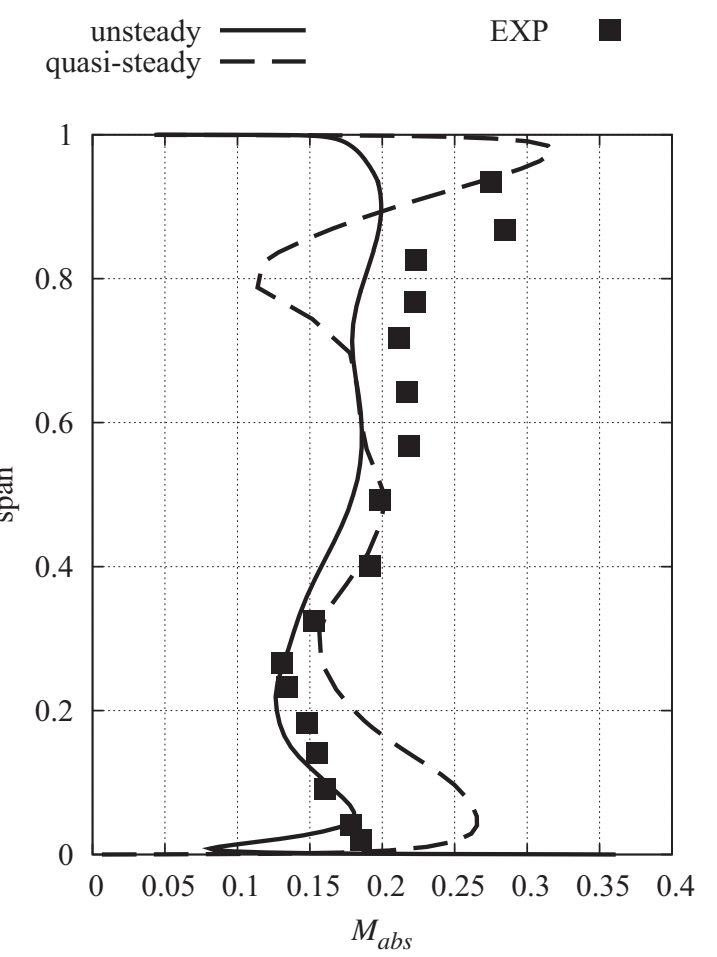

Fig. 13. Span-wise distribution of tbsolute Mach number in plane 3 .
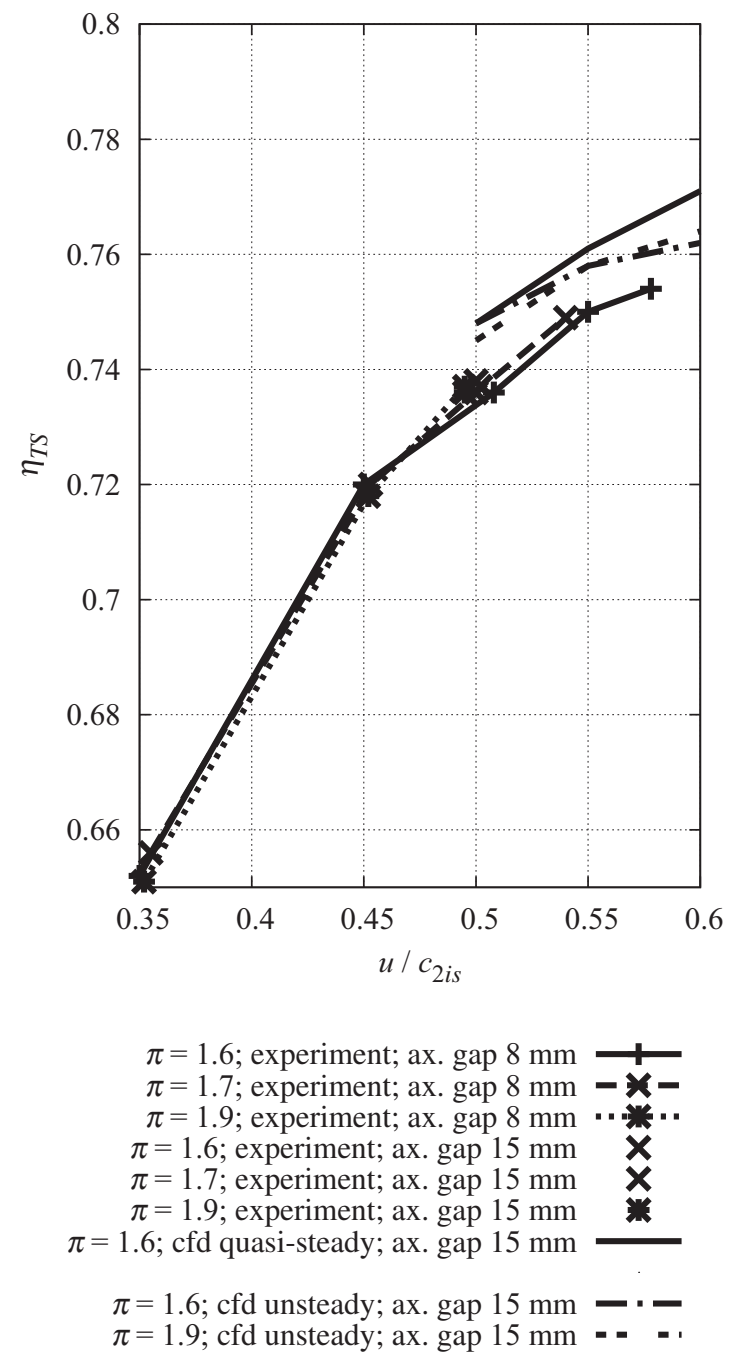

Fig. 14. Total-static efficiency in configuration with negative overlapping.

interaction of the rotor blades with the vortices behind the radial gap under the hub-end of the stator blades. Furthermore around thirty percent of the tip part of the span are affected by the vortices generated behind the radial gap above the tip-end of the rotor blades. This has negative impact on relatively low value of efficiency (see table 2 ).

\section{Future work}

Under the project, in which this work was created, new design of the axial turbine stage should be proposed. Currently, new design of stator blades is proposed and a background for design of new rotor blades is prepared. Based on acquired knowledge about properties of the prismatic stator blades with the radial gap, new design proposes nonprismatic blades with different profiles for hub- and tipsection. Moreover blades are equipped with hub-shroud which suppresses negative impact of the radial gap. For reduction of a production cost new stator blades is proposed as a ruled surface. Figure 16 shows shape of new non-prismatic stator blade with the hub-shroud in configuration with old prismatic rotor blade. 


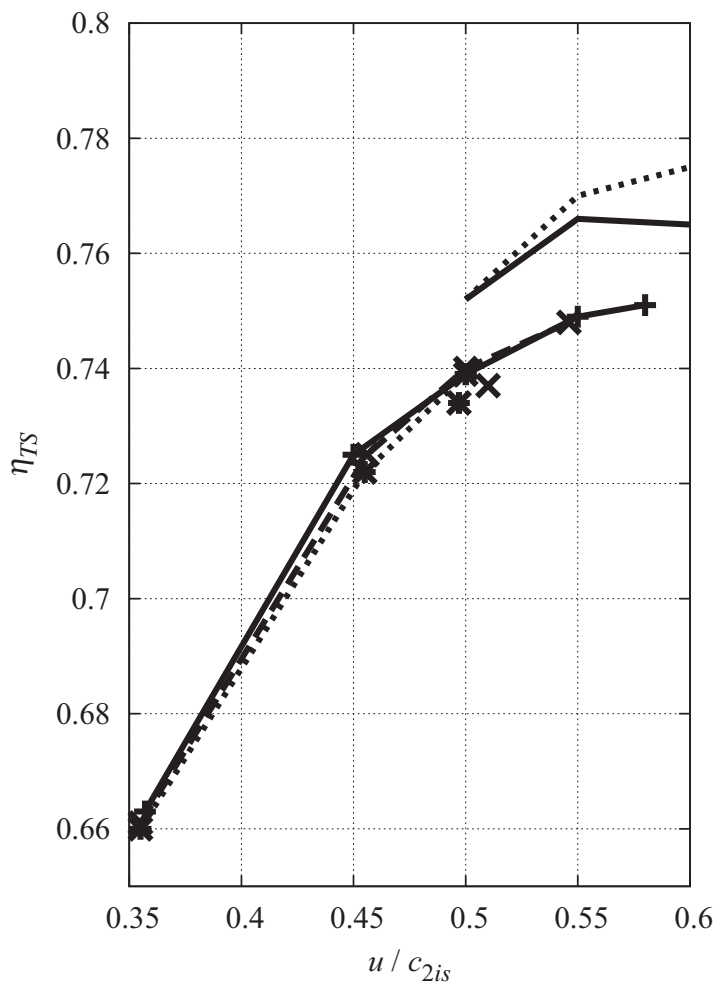

$$
\begin{array}{r}
\pi=1.6 \text {; experiment; ax. gap } 8 \mathrm{~mm} \\
\pi=1.7 \text {; experiment; ax. gap } 8 \mathrm{~mm} \\
\pi=1.9 \text {; experiment; ax. gap } 8 \mathrm{~mm} \\
\begin{aligned}
\pi=1.7 \text {; experiment; ax. gap } 15 \mathrm{~mm} \\
\pi=1.9 \text {; experiment; ax. gap } 15 \mathrm{~mm}
\end{aligned} \\
\pi=1.6 \text {; cfd quasi-steady; ax. gap } 15 \mathrm{~mm} \\
\pi=1.9 \text {; cfd quasi-steady; ax. gap } 15 \mathrm{~mm}
\end{array}
$$

Fig. 15. Total-static efficiency in configuration with positive overlapping.

Subsequently, new design of the rotor blades will be proposed.

Because the optimal velocity ratio is located out of range of the measured and computed regimes, it would be appropriate at least to compute some regimes at higher velocity ratio.

Because the main physical phenomenon which influences the efficiency in this kind of turbine stages is generating and interaction of large vortex structures, it would be better to use in simulation some turbulence model which is better suitable for this case (for example explicit algebraic Reynolds-stress model).

\section{Acknowledgement}

This work was supported by the grant project TA02021336 of the Technology Agency of the Czech Republic and by the Long-term Framework Advancement Plan provided by the Ministry of Industry and Trade of the Czech Republic.

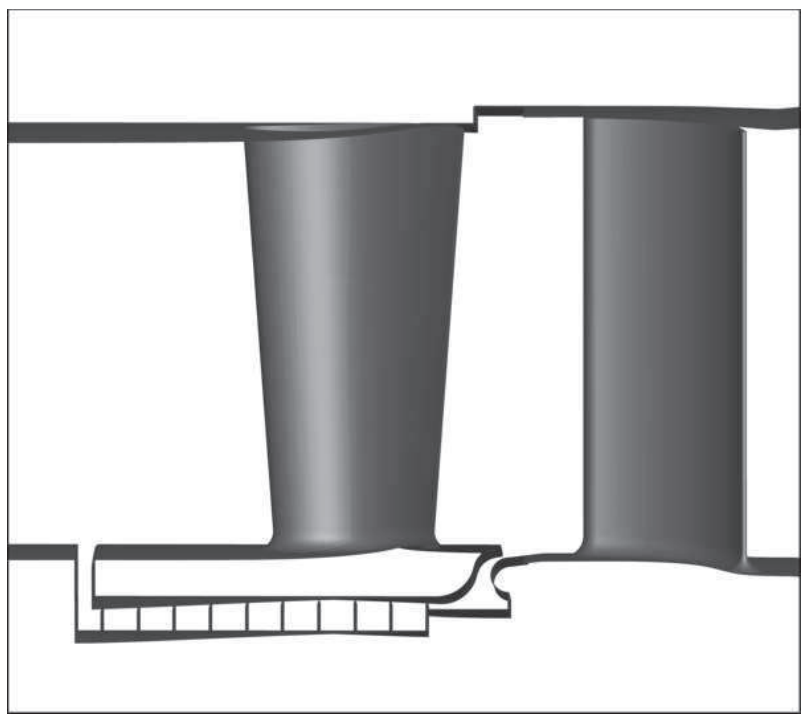

Fig. 16. Shape of new stator design.

\section{References}

1. V.A. Chernikov, Aerodyn. Characteristics of Therm. Turb. Stages, Leningrad, (1980) (in Russian)

2. D. Dey, C. Camci, ASME Paper No. 2001-GT-0484, (2001)

3. D.W. Thompson, P.I. King, D.C. Rabe, J. Turbomach. 120(3), (1998) 477-486

4. A. Huang, MSc Thesis, MTI, Massachusetts, (2011)

5. T.C. Booth, VKI Lecture Series 1985-05, VKI, (1985)

6. http://

www.isvav.cz/projectDetail.do?rowId=TA02021336

7. http://www.vzlu.cz/en/activities/ aerodynamics-wind-tunnel-testing/

a-wind-tunnel-for-turbo-machinery-purposes

8. T. Jelínek, M. Němec, Turbomachinery 2010, Plzeň, (2010) (in Czech)

9. M. Němec, T. Jelínek, J. Benetka, VZLU report R4939, Prague, (2010) (in Czech)

10. J. Lepicovsky, Proc. 18th Symp. Measuring Techniques in turbomachinery, Thessaloniki, (2006)

11. A. Pfau, J. Schlienger, A.I. Kalfas, R.S. Abhari, Proc. 16th Symp. Measuring Techniques in turbomachinery, Cambridge, (2002)

12. P. Kupferschmied, P. Köppel, W. Gizzi, C. Roduner, G. Gyarmathy, Measur. Sc. Technology 11, (2000) 10361054

13. J.C. Kok, AIAA Journal 38, No. 7, (2000)

14. P. Straka, Proc. Conf. Exp. Fluid Mech. 979-987, (2011)

15. P. Straka, Technical Report VZLÚ R-4910, Prague, (2010) (in Czech) 